\title{
Dynamic analysis of earthquake phenomena by means of pseudo phase plane
}

\author{
António M. Lopes • J.A. TenreiroMachado
}

\begin{abstract}
This paper analyses earthquake data in the perspective of dynamical systems and its Pseudo Phase Plane representation. The seismic data is collected from the Bulletin of the International Seismological Centre. The geological events are characterised by their magnitude and geographical location and described by means of time series of sequences of Dirac impulses. Fifty groups of data series are considered, according to the Flinn-Engdahl seismic regions of Earth. For each region, Pearson's correlation coefficient is used to find the optimal time delay for reconstructing the Pseudo Phase Plane. The Pseudo Phase Plane plots are then analysed and characterised.
\end{abstract}

\section{Keywords}

Pseudo Phase Plane - Pearson's correlation . Optimal time delay $\cdot$ Seismic event

\section{Introduction}

Earthquakes are associated to catastrophes that cause severe destruction and fatalities. These events manifest themselves by shaking and sometimes displacing ground. Moreover, earthquakes may trigger other potentially destructive events like tsunamis, landslides and volcanic activity [1-4]. Scientists consider that Earth' lithosphere is composed of seven or eight major plates and several minor plates $[5,6]$. The plates move slowly with respect to each other due to convection currents that exist in the denser asthenosphere region of the mantle, below the terrestrial lithosphere and crust $[7,8]$. Plate's motion can be (i) convergent, originating subduction zones and destroying lithosphere; (ii) divergent, creating lithosphere and usually forming rifts; and (iii) transform, mainly causing lateral motion, neither creating, nor destroying lithosphere. Neighbouring plates meet each other at fault zones and, when moving along the fault surfaces, exhibit friction and stick-slip behaviour [9-11]. Asperities between the plates may increase stress, leading to strain energy accumulation around the fault surface. When the stress is sufficiently high to break through the asperities, a sudden motion of the plates occurs, accompanied by energy release, causing an earthquake[12].

Over the last decades several models were proposed to describe seismic activity [13-20, 58-60]. In certain models, the asperities on the fault surfaces resemble fractals sliding over each other, explaining the fractal scaling behaviour that has been observed in 
earthquake phenomena [21]. Earthquakes are likely to come in clusters. A cluster is most probable to occur shortly after another cluster and a cluster of clusters soon after another cluster of clusters. Moreover, the tectonic plates form a complex system owing to interactions among faults [22-24]. An earthquake may not only release stress on the local fault, but can also change stress conditions on other faults. Tectonic plates motion and strain accumulation processes interact on a range of scales from a few millimetres to thousands of kilometres and loading rates are not uniform in time [24, 25]. Earthquakes unveil long range correlations and long memory characteristics [26], which are typical of fractional order systems [27]. Scale invariances manifest not only in the frequencies of aftershocks, but also in the magnitudefrequency distributions and in the spatial distribution of hypocentres. Complex correlations in space, time and magnitude are characterised by self-similarity and absence of characteristic length-scale, meaning that seismic parameters exhibit power-law (PL) behaviour $[28,29]$.

The distribution of the size of earthquakes follows the Guttenberg-Richter (GR) law, stating that the number of earthquakes with magnitude $M$ greater than a given value $m \in \mathbf{R}^{+}$is given by [30]

$\log _{10} N(m)=q-r \cdot m$

The parameter $r$ has regional variation, being in the range $r \in[0.8 ; 1.06]$ for small and $r \in[1.23 ; 1.54]$ for big earthquakes, and parameter $q$ is a measure of the regional level of seismicity [31].

Given the relationship between seismic released energy and the magnitude of an earthquake, another form in which the GR law can be expressed is

$N(\varepsilon) \approx \varepsilon^{-\gamma}$

where $N(\varepsilon)$ is the number of earthquakes, defined as previously but, in this case, for events which release energy greater than $\varepsilon \in \mathbf{R}^{+}$, and parameter $\gamma \in$ $[0.8 ; 1.05]$ varies according to the region seismicity.

The Omori law [32] gives the distribution of the number of aftershocks, $N$, with magnitude $M \geq m$, where $m$ denotes a given threshold, versus time $t$ :

$$
\frac{d N(t)}{d t}=t^{-p}
$$

The first member of Eq. (3) represents the rate of occurrence of aftershocks at time $t$ after the main shock.
Parameter $p$ is close to unity for tectonically active regions [33-35].

Earthquake phenomena have been studied in the perspective of complex systems and fractals. For example, in [36] a new model for earthquakes is proposed, given the observations of self-similarity in various length scales in the roughness of fractured solid surfaces. The authors demonstrate that the contact area distribution between two fractal surfaces follows a unique PL. In [37] the irregularity and complexity of earthquake ground motions is investigated from the perspective of nonlinear dynamics. Analysis based on chaotic dynamics theory and chaotic time series are suggested to examine the nonlinear characteristics of strong earthquake ground motions. The fractal geometry of various segments of the San Andreas Fault system was studied in [38], suggesting that differences between observed seismic activity might be attributed to differences in fault complexity and fractal dimensions. In [39] is suggested that Self-Organised Criticality (SOC) is relevant for understanding earthquakes as a relaxation mechanism that organises the terrestrial crust at both spatial and temporal levels. Some authors $[40,41]$ emphasise the relationships between complex systems, fractals and fractional calculus [42-45].

This paper addresses the earthquake phenomena in the perspective of dynamical systems analysis by means of the Phase Pseudo Plane (PPP) representation. The PPP has been used in the context of nonlinear dynamics where there is some lack of knowledge about the system [46]. PPP is a special case of Pseudo Phase Space, where the embedding space dimension is two. PPP has been used to assess complexity in genome data [47], to study musical sounds and expose hidden relationships among music styles [48], to study the evolution of stock indices [49], and to investigate the effects of impacts in robot dynamics [46].

In this paper, data collected from the Bulletin of the International Seismological Centre [50] is organised in 50 groups, according to the Flinn-Engdahl (F-E) seismic regions of Earth [51-53]. For each region, the seismic events are modelled as time sequences of Dirac impulses. This means giving a smaller importance to the short period, and focusing on the long range dynamics revealed by the time series. Pearson's correlation is used to compare every signal with its time delayed version. The optimal time delay (in a per-

spective to be defined) is obtained and adopted in the PPP two dimensional chart, characterizing the time series dynamics. 
Fig. 1 Geographical location of seismic events, according to the ISC Bulletin (events with magnitude $M \geq 2$ ). The blue lines represent the borders of the F-E regions. The time period of reference is 1962-2011

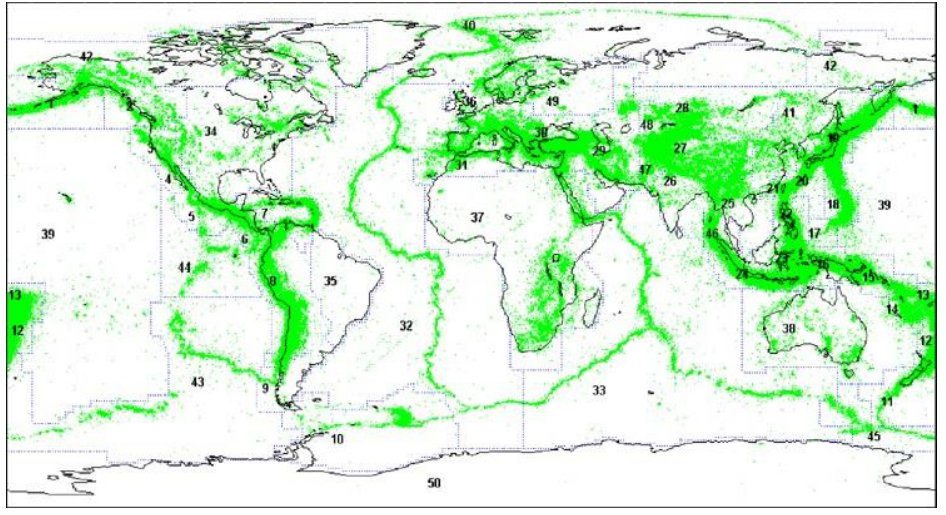

Bearing these ideas in mind, this paper is organised as follows. Section 2 describes the experimental dataset used in this study. Section 3 introduces the PPP technique and the main concepts involved. Section 4 formulates the framework of the analysis of earthquakes in the perspective of PPP. Finally, Sect. 5 outlines the main conclusions.

\section{Dataset and time-domain analysis}

Data compiled by the International Seismological Centre (ISC), available online at http://www.isc.ac.uk/ [57], is used in this study. The ISC Bulletin contains seismic events since 1904, relying on data collected by more than 17000 seismic stations located worldwide.

Each data record contains information about date and time, geographic location and magnitude of the events. Occurrences with magnitude in the interval

$M \in[-2.1,9.2]$, in a logarithm scale consistent with the local magnitude or Richter scale, are available [57].

Regarding the complete catalogue, important differences can be noted when comparing both the first and second periods of registers, each of about half a century. For the first period, the number of records is remarkable smaller and lower magnitude events are scarce. This may be justified by the technological constraints associated to the instrumentation available in the early decades of the twentieth century. To prevent bias errors, in our study the 50-year period from 1962 up to 2011 is processed. Moreover, we consider just the events with magnitude $M_{k} \geq 2$. Lower magnitude events are often due to man-triggered explosions and do not correspond to tectonic (natural) occurrences.
Seismic activity is far from being uniform across the Earth. Figure 1 shows the location of seismic events satisfying the conditions stated above. It can be seen that most seismic activity occurs in three large regions [54]: (i) the Circum-Pacific belt (also known as "Ring of Fire") which extends from Chile, northward along the South American coast through Central America, Mexico, the West Coast of the United States, and the southern part of Alaska, through the Aleutian Islands to Japan, the Philippine Islands, New Guinea, the island groups of the Southwest Pacific, and to New Zealand (about $90 \%$ of the world's earthquakes occur in this area); (ii) the second most seismic region (representing approximately $6 \%$ of all earthquakes) is the Alpide belt, which extends from the Mediterranean region, eastward through Turkey, Iran, and northern India; (iii) the firth major region is the submerged midAtlantic Ridge. Figure 1 also depicts F-E regions of Earth. These are often used by seismologists to locate earthquakes [51-53] and are listed in Table 1. The number of events with magnitude $M_{k} \geq 2$, observed during the period of analyses, and the maximum and average magnitudes, are also shown for every region.

As said before, the events belonging to a given F-E region $i(i=1, \ldots, 50)$ are represented by the timedomain signal:

$$
x_{\mathrm{FE}_{i}}(t)=\sum_{k=1}^{T} M_{k} \delta\left(t-t_{k}\right)
$$

meaning that the seismic events are simply modelled as sequences of Dirac impulses, $M_{k} \delta\left(t-t_{k}\right)$, where $M_{k}$ represents the magnitude, $t_{k}$ is the time of the occurrence, parameter $t$ represents time and $T$ is the total time period of study, both expressed in second [55]. 
Table 1 Flinn-Engdahl regions of Earth and characterisation of the events, with magnitude $M_{k} \geq 2$, observed in the period 1962-2011

\begin{tabular}{|c|c|c|c|c|}
\hline F-E region & F-E name & Number of events & Maximum magnitude & Average magnitude \\
\hline 1 & Alaska-Aleutan arc & 38966 & 8.0 & 3.7 \\
\hline 2 & Southeastern Alaska to Washington & 17180 & 7.1 & 2.7 \\
\hline 3 & Oregon, California and Nevada & 23697 & 7.6 & 3.0 \\
\hline 4 & Baja California and Gulf of California & 5074 & 7.2 & 3.2 \\
\hline 5 & Mexico-Guatemala area & 29964 & 7.9 & 3.9 \\
\hline 6 & Central America & 20423 & 7.5 & 3.8 \\
\hline 7 & Caribbean loop & 44902 & 7.3 & 3.1 \\
\hline 8 & Andean South America & 77723 & 8.5 & 3.6 \\
\hline 9 & Extreme South America & 2424 & 6.3 & 3.3 \\
\hline 10 & Southern Antilles & 6101 & 7.5 & 4.4 \\
\hline 11 & New Zealand region & 56800 & 8.1 & 3.2 \\
\hline 12 & Kermadec-Tonga-Samoa Basinarea & 49888 & 8.1 & 4.2 \\
\hline 13 & Fiji Islands area & 23701 & 7.2 & 4.0 \\
\hline 14 & Vanuatu Islands & 28907 & 7.9 & 4.1 \\
\hline 15 & Bismarck and Solomon Islands & 28881 & 8.0 & 4.1 \\
\hline 16 & New Guinea & 24949 & 7.8 & 4.0 \\
\hline 17 & Caroline Islands area & 5013 & 7.0 & 4.1 \\
\hline 18 & Guam to Japan & 33499 & 7.5 & 3.7 \\
\hline 19 & Japan-Kuril Islands-Kamchatka Peninsula & 284565 & 8.3 & 2.9 \\
\hline 20 & Southwestern Japan and Ryukyu Islands & 101055 & 7.4 & 2.6 \\
\hline 21 & Taiwan area & 170943 & 7.9 & 2.6 \\
\hline 22 & Philippine Islands & 31130 & 8.4 & 3.9 \\
\hline 23 & Borneo-Sulawesi & 34252 & 7.5 & 4.0 \\
\hline 24 & Sunda arc & 46414 & 8.4 & 4.0 \\
\hline 25 & Myanmar and Southeast Asia & 6154 & 7.4 & 3.6 \\
\hline 26 & India-Xizang-Sichuan-Yunnan & 19620 & 8.0 & 3.4 \\
\hline 27 & Southern Xinjiang to Gansu & 11394 & 8.0 & 3.4 \\
\hline 28 & Lake Issyk-Kul to Lake Baykal & 24304 & 7.4 & 3.0 \\
\hline 29 & Western Asia & 18848 & 8.1 & 3.5 \\
\hline 30 & Middle East-Crimea-Eastern Balkans & 192461 & 8.4 & 2.9 \\
\hline 31 & Western Mediterranean area & 80168 & 7.2 & 2.6 \\
\hline 32 & Atlantic Ocean & 25889 & 7.0 & 3.5 \\
\hline 33 & Indian Ocean & 12820 & 7.7 & 4.1 \\
\hline 34 & Eastern North America & 14399 & 7.3 & 2.7 \\
\hline 35 & Eastern South America & 67 & 5.7 & 4.3 \\
\hline 36 & Northwestern Europe & 27071 & 5.9 & 2.4 \\
\hline 37 & Africa & 34510 & 7.4 & 3.0 \\
\hline 38 & Australia & 5531 & 6.5 & 3.0 \\
\hline 39 & Pacific Basin & 2085 & 7.0 & 3.9 \\
\hline 40 & Arctic zone & 12009 & 6.9 & 3.1 \\
\hline 41 & Eastern Asia & 8911 & 7.8 & 3.4 \\
\hline 42 & Northeast. Asia, North. Alaska to Greenland & 6602 & 7.6 & 3.2 \\
\hline 43 & Southeastern and Antarctic Pacific Ocean & 6943 & 7.1 & 4.3 \\
\hline 44 & Galápagos Islands area & 2351 & 6.4 & 4.2 \\
\hline 45 & Macquarie loop & 1743 & 7.8 & 4.3 \\
\hline 46 & Andaman Islands to Sumatra & 20755 & 9.2 & 4.0 \\
\hline 47 & Baluchistan & 4099 & 7.6 & 3.9 \\
\hline 48 & Hindu Kush and Pamir area & 32811 & 7.3 & 3.3 \\
\hline 49 & Northern Eurasia & 5751 & 5.9 & 2.4 \\
\hline 50 & Antarctica & 64 & 5.5 & 4.0 \\
\hline
\end{tabular}


Fig. 2 Time-domain signal $x_{\mathrm{FE}_{1}}(t)$, corresponding to Alaska-Aleutan arc. The events with magnitude $M_{k} \geq 2$ and period of time 1962 2011 are considered

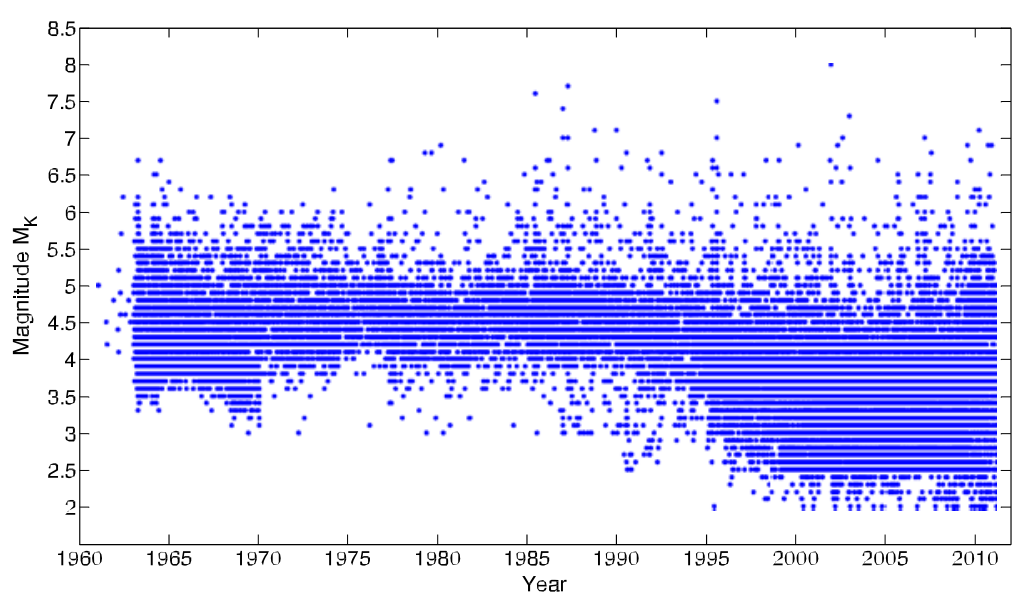

$$
\begin{aligned}
& Y_{\mathrm{FE}_{i}}(t)=\int_{0}^{t} y_{\mathrm{FE}_{i}}(u) d u \\
& r_{i j}=\frac{\sum_{t-1}^{T}\left[Y_{\mathrm{FE}_{i}}(t)-\bar{Y}_{\mathrm{FE}_{i}}\right] \cdot\left[Y_{\mathrm{FE}_{j}}(t)-\bar{Y}_{\left.\mathrm{FE}_{j}\right]}\right]}{\sqrt{\sum_{t-1}^{T}\left[Y_{\mathrm{FF}_{i}}(t)-\bar{Y}_{\left.\mathrm{FE}_{i}\right]^{2}}\right.} \cdot \sqrt{\sum_{t-1}^{T}\left[Y_{\mathrm{FF}_{j}}(t)-\bar{Y}_{\mathrm{FE}_{j}}\right]^{2}}}, \\
& i, j=1, \ldots, 50
\end{aligned}
$$

particular single event. In our methodology $x_{\mathrm{FE}_{i}}(t)$ are descriptive signals of the earthquake dynamics. As an illustrative example, Fig. 2 represents $x_{\mathrm{FE}_{1}}(t)$, corresponding to F-E region Alaska-Aleutan arc, revealing noisy and chaotic-like characteristics, typically seen in many nonlinear and complex phenomena. The quantisation levels observed in the magnitudes $M_{k}$ are due to the fact that the data records are represented with limited resolution. It should be noted that Alaska is the most seismically active state in the U.S. and has registered the second-largest earthquake ever recorded on Earth.

It should be noted again that magnitudes $M_{k}<2$ are not considered because natural events are often polluted with man-produced occurrences. This somewhat limits accuracy of the study, but the alternative option is impractical.

In order to reveal eventual relationships between the F-E regions, we adopted Pearson's correlation coefficient, $r_{i j}$. First, the signals $y_{\mathrm{FE}_{i}}(t)$ are computed using the magnitudes of the events expressed in linear units:

$$
y_{\mathrm{FE}_{i}}(t)=\sum_{k=1}^{T} A_{k} \delta\left(t-t_{k}\right)
$$

where $A_{k}=10^{M_{k}}$. Second, the time integrals, $Y_{\mathrm{FE}_{i}}(t)$, are determined and the correlation coefficients $r_{i j}$ calculated: where $Y_{\mathrm{FE}_{i}}(t)$ and $Y_{\mathrm{FE}_{j}}(t)$ represent the $(i, j)$ F-E region pairs and $\bar{Y}_{\mathrm{FE}_{i}}, \bar{Y}_{\mathrm{FE}_{j}}$ denote the arithmetic mean values of $Y_{\mathrm{FE}_{i}}(t)$ and $Y_{\mathrm{FE}_{j}}(t)$, over period $T$, respectively.

For the F-E region Alaska-Aleutan arc, is depicted in Fig. 3 the integral $Y_{\mathrm{FE}_{1}}(t)$ as a function of time. The observed larger discontinuities reflect the presence of time periods with remarkable higher seismic activity, namely years 1986, 1988, 1996, 2002 and 2003. In all these years large earthquakes were recorded. In particular, we can mention the 1986 Andreanof Islands and the 1996 Delarof Islands earthquakes, which belong to the larger historic events.

It is possible to use either logarithm, $M_{k}$, or linear, $A_{k}$, units. Experiments demonstrated that expressing the magnitudes of the seismic occurrences in linear scales, gives clear results in terms of visibility and readability of the plots. On the other hand, $y \mathrm{FE}_{i}(t)$ and $x_{\mathrm{FE}_{i}}(t)$ are sparse vectors, populated primarily with zeros, when adopting a time scale resolution of one second. Computing time correlations using those signals could, in the limit, result in $r_{i j}$ equal to zero, because no two distinct events are likely to occur at identical time instants (at the one-second scale resolution), hiding important characteristics of the real data.

Figure 4 depicts Pearson's correlation coefficient, $r_{i j}$, defined in (7). It can be noted that F-E regions 
Fig. 3 Integral $Y_{\mathrm{FE}_{1}}(t)$, corresponding to F-E region AlaskaAleutan arc, depicted as a function of time

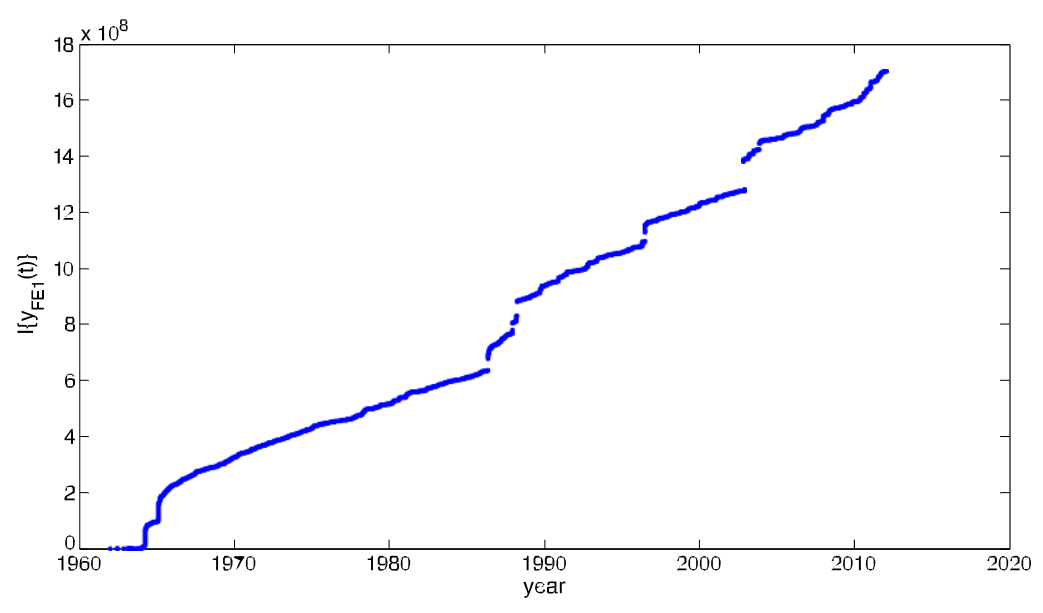

Fig. 4 Pearson's correlation coefficient, $r_{i j}$, between every pair of F-E regions. The events with magnitude $M_{k} \geq 2$ and period of analysis 1962 2011 are considered

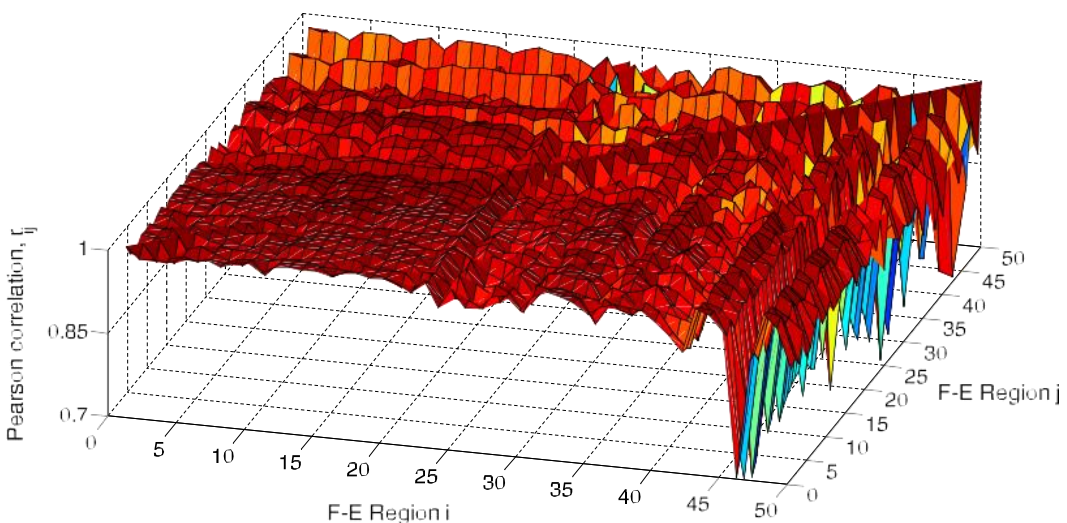

$\{1-22\}$ are strongly correlated and $r_{i j}$ varies smoothly. For the other regions the index $r_{i j}$ is more "noisy". The groups $\{24,26,27,41\}$ and $\{30,35,40,45\}$ can be identified as being weakly correlated with most zones; for group $\{42,47,50\}$ correlation is small; and zone $\{46\}$ is almost uncorrelated with the rest.

To complement the analysis, we adopt a standard clustering algorithm for comparing results. The phylogenetic analysis open source software PHYLIP (http://evolution.genetics.washington.edu/phylip.html) is adopted.

For this purpose we calculate matrix $\mathbf{D}=\left[d_{i j}\right]$, where $d_{i j}=1-r_{i j}$. The corresponding circular phylogram is generated by successive (agglomerative) clustering and represented in Fig. 5. The leaves represent F-E regions. An average-linkage method was used to generate the tree. The clusters mentioned above can now be clearly identified, being in accordance to Fig. 4.

\section{Pseudo Phase Plane}

In dynamical systems the Pseudo Phase Space (PPS) reconstruction is often used to mitigate the lack of information about the system [46, 61]. A representation of the system dynamics in a higher dimensional space is made possible by taking a smaller sample of signals representing measurements of the system time history. The PPP is justified by Takens' embedding theorem [56], which states that if a time series is one component of an attractor that can be represented by a smooth $d$-dimensional manifold, then the topological properties of the signal are equivalent to those of the embedding formed by the $n$-dimensional phase space vectors:

$$
u(t)=[s(t) s(t+\tau) s(t+2 \tau) \cdots s(t+(n-1) \tau)]
$$




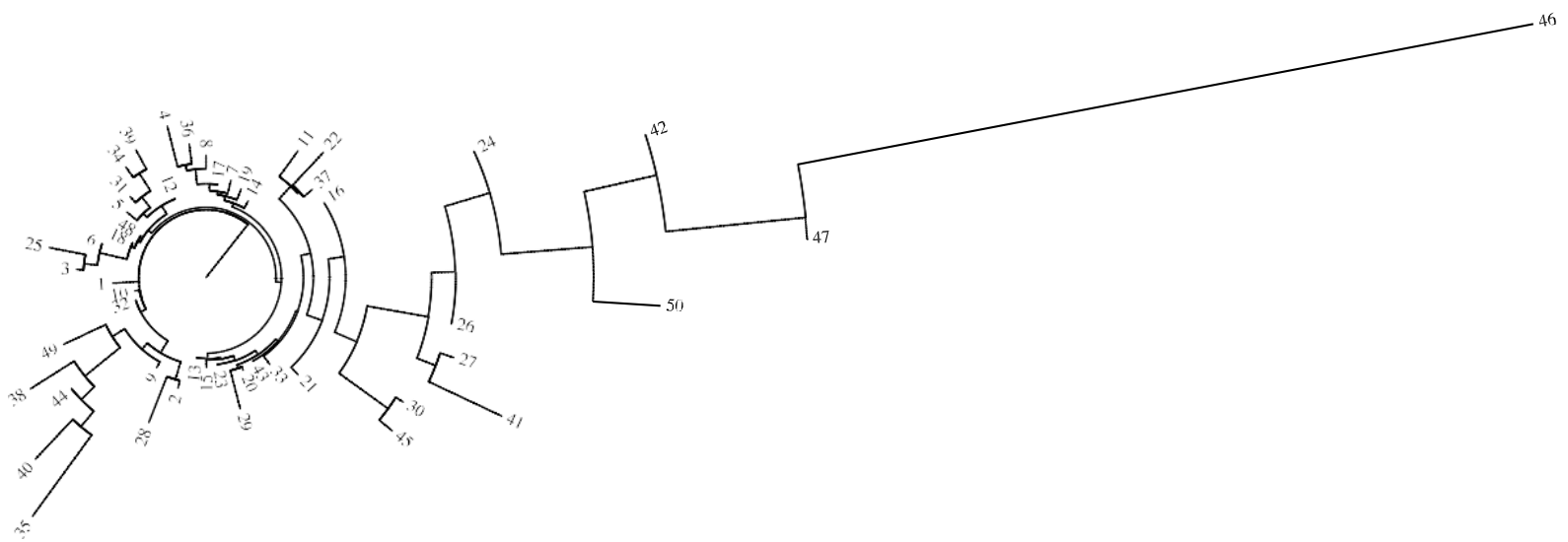

Fig. 5 Circular phylogram based on index $d_{i j}=1-r_{i j}$, used to compare F-E regions. Are considered events with magnitude $M_{k} \geq 2$ and period of analysis is 1962-2011

where $n>2 d+1,\{d, n\} \in \mathbf{N}, \tau \in \mathbf{R}^{+}$. Parameters $\tau$ and $n$ represent the time delay and embedding dimension, respectively. The vector $u(t)$ is usually plotted in a $n$-dimensional graph, forming a path in the PPS, that depends on the values of $\tau$ and $n$. Usually, $n=3$ or $n=2$ are chosen in order to facilitate the interpretation of the graphs. In the latter case a two-dimensional time delay space is obtained and the PPS degenerates in the PPP. In this case, the vector $u(t)=[s(t) s(t+\tau)]$ is related to the model given by the state vectors $\left[s(t) s^{*}(t)\right]$. The PPP produced by $u(t)$ is expected to allow conclusions about the system dynamics [10].

A key issue with PPS is the choice of time delay $\tau$. Intuitively, choosing $\tau$ too small, will result in time series $s(t)$ and $s(t+\tau)$ close to each other and, virtually, undistinguishable. From a practical point of view, they do not provide two independent coordinates. On the other hand, choosing $\tau$ very large would lead to series $s(t)$ and $s(t+\tau)$ almost independent of each other providing totally unrelated directions. This option is of no practical use since the intersection pair of coordinates would vanish. Therefore, some compromise is needed.

There have been several proposals for choosing the optimal time delay, $\tau_{m}$, mainly based on the behaviour of the autocorrelation function, where a time series is correlated to its own delayed image. The earliest time at which the autocorrelation function decreases below a certain percentage of its initial value, or has a point of inflection, has been used as a criterion to obtain the delay $\tau_{m}$. These definitions seek to find times where linear correlations between different points in the time series are negligible, but they do not rule out the possibility of more general correlations [49]. The mutual information has also been used when autocorrelation reveals difficulties to deal with nonlinear relations. One possible criterion for choosing $\tau_{m}$ is to consider the value corresponding to the first local minimum of the mutual information. The mutual information is a measure of how much information can be predicted about one time series point giving full information about the other [48]. The values of $\tau_{m}$ at which the mutual information has a local minimum are equivalent to the values of $\tau_{m}$ at which the logarithm of the correlation sum has a local minimum. It is not clear which method, if any, is superior for all topological properties. However, optimal values based on the behaviour of the autocorrelation function are the easier to compute [13].

\section{Pseudo Phase Plane analysis of earthquake phenomena}

In this section, we use the PPP technique to analyse the 50 F-E regions. Firstly, the autocorrelation of each signal $Y_{\mathrm{FE}_{i}}(t)$ is calculated. The Pearson's correlation coefficient is used to correlate $Y_{\mathrm{FE}_{i}}(t)$ with its time delayed version $Y_{\mathrm{FE}_{i}}(t-\tau)$ and the optimal time delays, $\tau_{m_{i}}$, are computed. In a second step, the PPP is reconstructed and analysed. 
Fig. 6 Evolution of $r_{11}(\tau)$, corresponding to F-E region Alaska-Aleutan arc. The optimum time delay is $\tau_{m_{1}}=13$ years

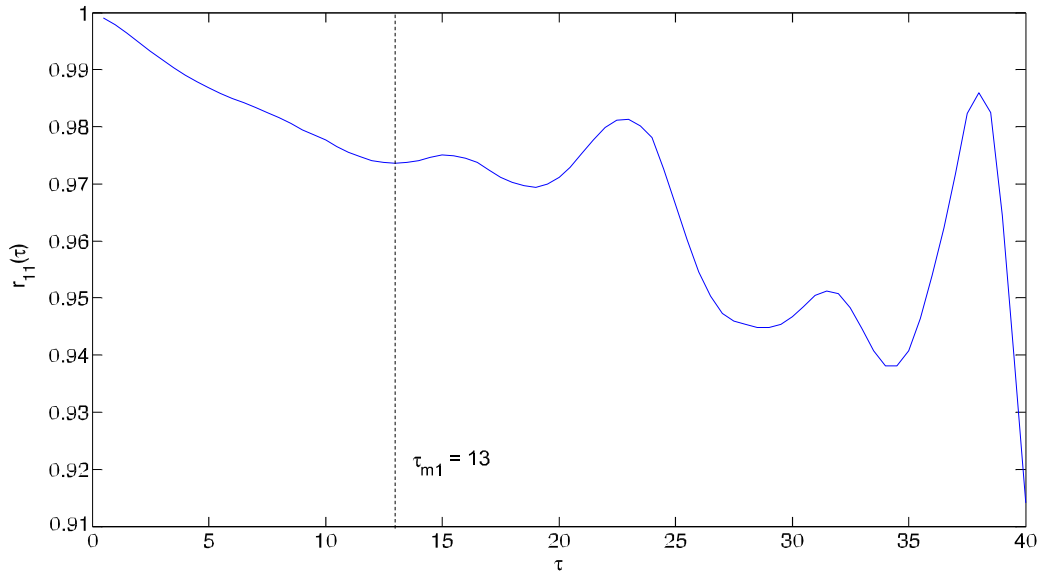

Fig. 7 Optimum time delays for the whole set of F-E zones. The numbers represent $\tau_{m_{i}}$ for each region and the colours are set according to the shown colourmap

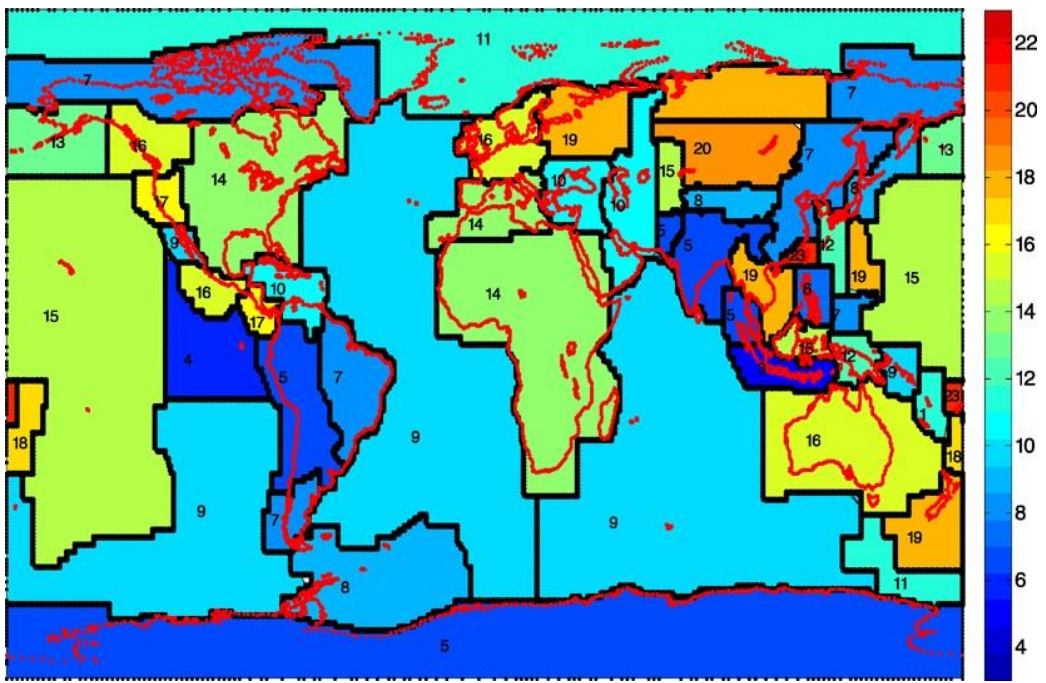

where the optimum time delay yields $\tau_{m_{1}}=13$ years. Similar graphs are obtained for all regions. As can be seen in Fig. 7, the optimum time delays vary significantly for the whole set of F-E regions. The smaller value is $\tau_{m}=3$ years for F-E region $\{24\}$ (Sunda $\operatorname{arc}$ ), and the larger value is $\tau_{m}=23$ years, for the F-E re-

gions $\{5,13,21\}$ (Mexico-Guatemala area, Fiji Islands area and Taiwan area, respectively). The numbers in Fig. 7 represent $\tau_{m_{i}}$ for each region and the colours are set according to a colourmap dependent on $\tau_{m}$.

\subsection{Pseudo Phase Plane reconstruction and analysis}

In this subsection we compute all signals, $Y_{\mathrm{FE}_{i}}(t)$, representing the F-E regions and the PPP graphs are analysed. 
Fig. 8 PPP depicting $Y_{\mathrm{FE}_{1}}\left(t-\tau_{m_{1}}\right)$ versus $Y_{\mathrm{FE}_{1}}(t)$ for F-E region Alaska-Aleutan arc:

$\tau_{m_{1}}=13$
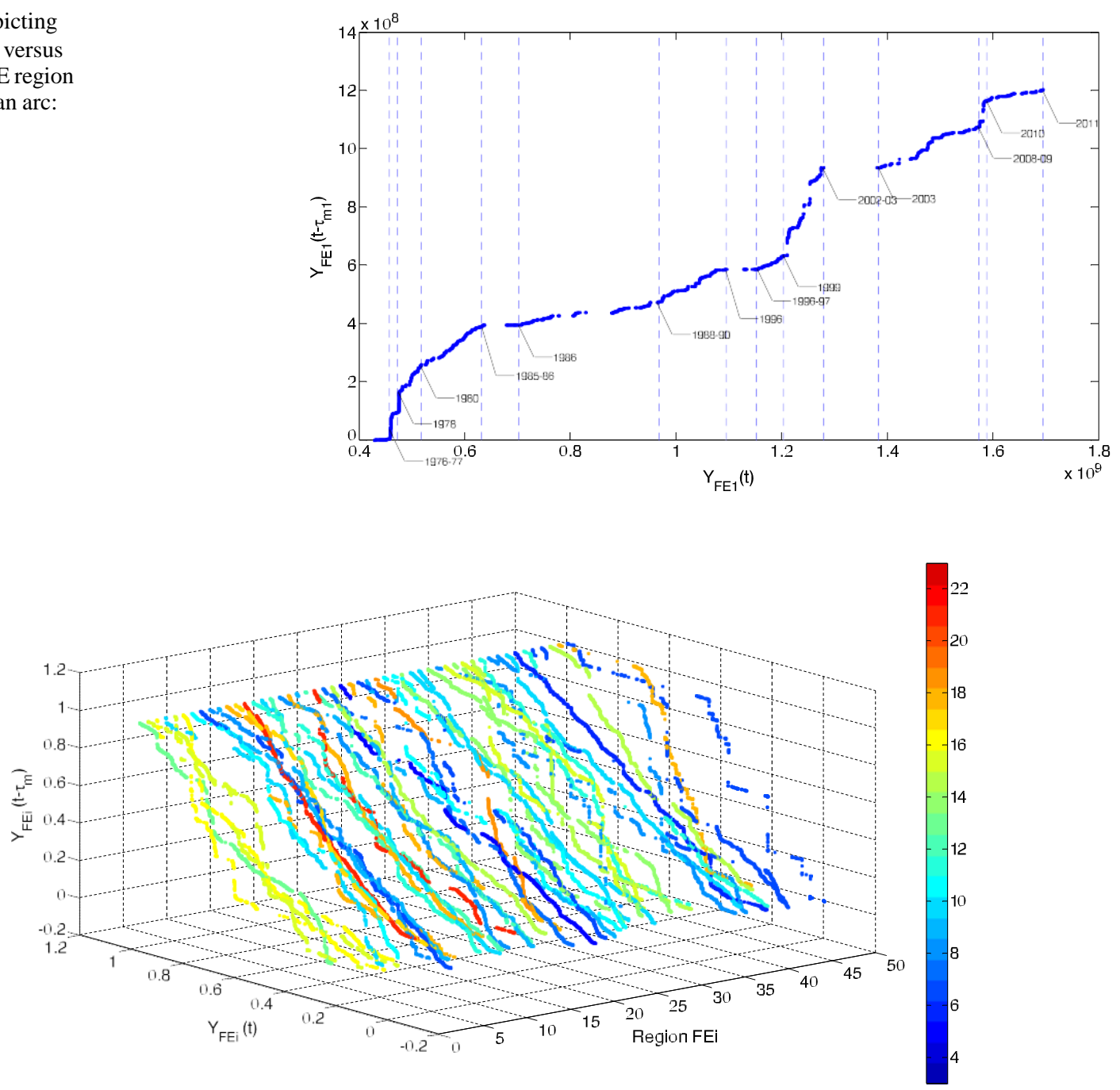

Fig. 9 PPP depicting $Y_{\mathrm{FE}_{i}}\left(t-\tau_{m_{i}}\right)$ versus $Y_{\mathrm{FE}_{i}}(t)$ for all F-E regions. The colours correspond to those used in Fig. 7

Figure 8 depicts, for example, the PPP of $Y_{\mathrm{FE}_{1}}(t-$ $\left.\tau_{m_{1}}\right)$ versus $Y_{\mathrm{FE}_{1}}(t)$. The locus has different grades of "granularity" since for some parts we have many points close to each other, while in other parts we verify sudden changes and only a few points. The slope of the trace reflects the rate of earthquakes. Therefore, a smaller/larger slope for instant $t$ corresponds to a higher/lower rate of Dirac impulses with respect to instant $t-\tau_{m_{1}}$. For the F-E region Alaska-Aleutan arc, 14 slices emerge corresponding to the time periods in the labels (Fig. 8). Higher seismic activity periods identified previously in Figs. 4 and 5, namely, years 1986, 1988, 1996, 2002 and
2003 are confirmed. Moreover, other periods, as 19761977, 1978, 1980, 1985-1986, 1988-1990, 19961997, 1999, and 2008-2009 are revealed clearly by the PPP.

The 50 PPP of all F-E regions are calculated and normalised in the sense that their $x$ - and $y$-axis are scaled to the interval between o and 1. In Fig. 9, the PPP normalised curves are shown. We use the same colourmap as adopted previously in order to ease the comparison among regions and to view the relationships with parameter $\tau_{m_{i}}$.

We now calculate the $50 \times 50$ matrix $\mathbf{E}=\left[e_{i j}\right]$ of distances, based on the Euclidean distance, $e_{i j}$, be- 


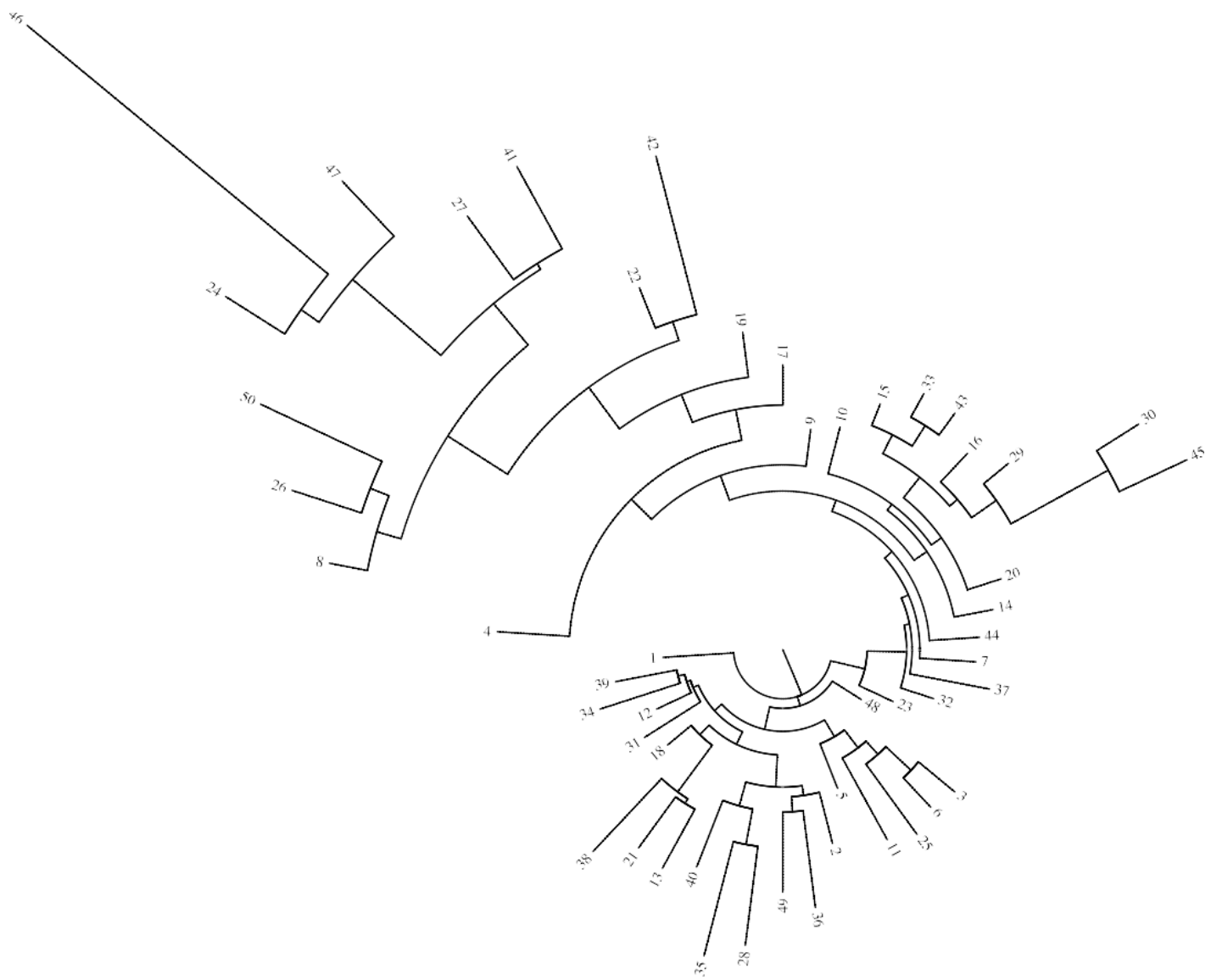

Fig. 10 Circular phylogram based on Euclidean distance index $e_{i j}$ used to compare all F-E regions PPP curves

tween the PPP curves, defined as

$$
\begin{aligned}
e_{i j}= & \left(\sum_{t=1}^{T}\left[Y_{\mathrm{FE}_{i}}(t)-Y_{\mathrm{FE}_{j}}(t)\right]^{2}\right. \\
& \left.+\left[Y_{\mathrm{FE}_{i}}\left(t-\tau_{m_{i}}\right)-Y_{\mathrm{FE}_{j}}\left(t-\tau_{m_{j}}\right)\right]^{2}\right)^{\frac{1}{2}}
\end{aligned}
$$

Based on matrix $\mathbf{E}$ we can draw the circular phylogram shown in Fig. 10. The F-E regions groups $\{22$, $42\}$ and $\{30,45\} ;\{8,26,50\}$ and $\{27,41\} ;\{27,47\}$ and $\{46\}$ form clusters increasingly different from the rest.

Comparing the circular phylograms of Figs. 5 and 10 , coming from different mathematical tools, we verify a considerable coincidence of results, namely an outer group of objects (e.g., $\{46\} ;\{24,47\} ;\{8,26$, $50,27,41\} ;\{42,22\} ;\{30,45\})$ and a denser in- ner group of items. This means that the smaller outer group is very different from the rest, while the larger inner group share considerable similarities.

A more detailed analysis of the pros and cons of (7) over (10) needs to be further explored based on other evidences and practical knowledge in the field. The proposed indices can help in establishing a more solid methodology for understanding the overall complex dynamics of the Earth lithosphere.

\section{Conclusion}

Two methods were proposed to measure Earth's seismic activity based on the time series of events. A first method based on Pearson's correlation coefficient and a second method based on the PPP plot. In both cases it was considered the time integral of the time series of Dirac impulses, where each impulse represents an 
earthquake. The two methods lead to distance matrices that can be studied by modern visualisation algorithms borrowed from distinct scientific areas. In the present case it was decided to adopt phylograms, usual in the area of genetics, providing charts that capture all information but reasonably easy to interpret. Different measures lead to slightly distinct phylograms. Therefore, the decision for a given measure will depend on the verification and validation by professionals based on field evidences.

\section{References}

1. Ghobarah, A., Saatcioglu, M., Nistor, I.: The impact of the 26 December 2004 earthquake and tsunami on structures and infrastructure. Eng. Struct. 28, 312-326 (2006)

2. Marano, K., Wald, D., Allen, T.: Global earthquake casualties due to secondary effects: a quantitative analysis for improving rapid loss analyses. Nat. Hazards 52, 319-328 (2010)

3. Lee, S., Davidson, R., Ohnishi, N., Scawthorn, C.: Fire following earthquake - reviewing the state-of-the-art modelling. Earthq. Spectra 24, 933-967 (2008)

4. Bird, J.F., Bommer, J.J.: Earthquake losses due to ground failure. Eng. Geol. 75, 147-179 (2004)

5. Anderson, D.L.: How many plates? Geology 30, 411-414 (2002)

6. Sornette, D., Pisarenko, V.: Fractal plate tectonics. Geophys. Res. Lett. (2003). doi:10.1029/2002GL015043

7. Stadler, G., Gurnis, M., Burstedde, C., Wilcox, L.C., Alisic, L., Ghattas, O.: The dynamics of plate tectonics and mantle flow: from local to global scales. Science 329, 1033-1038 (2010)

8. Bellahsen, N., Faccenna, C., Funiciello, F.: Dynamics of subduction and plate motion in laboratory experiments: insights into the "plate tectonics" behavior of the Earth. J. Geophys. Res. (2005). doi:10.1029/2004JB002999

9. Bhattacharya, P., Chakrabarti, B.K., Kamal: A fractal model of earthquake occurrence: theory, simulations and comparisons with the aftershock data. J. Phys. Conf. Ser. 319, 012004 (2011)

10. Carlson, J.M., Langer, J.S., Shaw, B.E.: Dynamics of earthquake faults. Rev. Mod. Phys. 66, 657-670 (1994)

11. De Rubeis, V., Hallgass, R., Loreto, V., Paladin, G., Pietronero, L., Tosi, P.: Self-affine asperity model for earthquakes. Phys. Rev. Lett. 76, 2599-2602 (1996)

12. Lopes, A.M., Tenreiro Machado, J.A., Pinto, C.M.A., Galhano, A.M.S.F.: Fractional dynamics and MDS visualization of earthquake phenomena. Comput. Math. Appl. (2013). doi:10.1016/j.camwa.2013.02.003

13. Sobolev, G.A.: Seismicity dynamics and earthquake predictability. Nat. Hazards Earth Syst. Sci. 11, 445-458 (2011)

14. Bak, P., Tang, S.: Earthquakes as self-organized critical phenomenon. J. Geophys. Res., Solid Earth 94, 1563515637 (1989)
15. Chelidze, T., Kolesnikov, Y., Matcharashvili, T.: Seismological criticality concept and percolation model of fracture. Geophys. J. Int. 164, 125-136 (2006)

16. Hainzl, S., Zoller, G., Kurths, J., Zschau, J.: Seismic quiescence as an indicator for large earthquakes in a system of self-organized criticality. Geophys. Res. Lett. 27, 597-600 (2000)

17. Harris, R.A., Day, S.M.: Dynamics of fault interaction: parallel strike-slip faults. J. Geophys. Res., Solid Earth 98, 4461-4472 (1993)

18. Stakhovsky, I.R.: Self-similar seismogenic structure of the crust: a review of the problem and a mathematical model. Izvest. Phys. Solid Earth 43, 1012-1023 (2007)

19. Sobolev, G., Spetzler, H., Koltsov, A., Chelidze, T.: An experimental study of triggered stick-slip. Pure Appl. Geophys. 140, 79-94 (1993)

20. Scholz, C.H.: Large earthquake triggering, clustering, and the synchronization of faults. Bull. Seismol. Soc. Am. 100, 901-909 (2010)

21. Hallgass, R., Loreto, V., Mazzella, O., Paladin, G., Pietronero, L.: Earthquake statistics and fractal faults. Phys. Rev. E 56, 1346-1356 (1997)

22. Sarlis, N.V., Christopoulos, S.-R.G.: Natural time analysis of the centennial earthquake catalog. Chaos 22, 023123 (2012)

23. Turcotte, D.L., Malamud, B.D.: Earthquakes as a complex system. Int. Geophys. Ser. 81, 209-227 (2002)

24. Kanamori, H., Brodsky, E.: The physics of earthquakes. Rep. Prog. Phys. 67, 1429-1496 (2004)

25. Stein, S., Liu, M., Calais, E., Li, Q.: Mid-continent earthquakes as a complex system. Seismol. Res. Lett. 80, 551553 (2009)

26. Lennartz, S., Livina, V.N., Bunde, A., Havlin, S.: Longterm memory in earthquakes and the distribution of interoccurrence times. Europhys. Lett. 81, 69001 (2008)

27. El-Misiery, A.E.M., Ahmed, E.: On a fractional model for earthquakes. Appl. Math. Comput. 178, 207-211 (2006)

28. Pinto, C.M.A., Lopes, A.M., Tenreiro Machado, J.A.: A review of power laws in real life phenomena. Commun. Nonlinear Sci. Numer. Simul. 17, 3558-3578 (2012)

29. Sornette, D., Knopoff, L., Kagan, Y., Vanneste, C.: Rankordering statistics of extreme events: application to the distribution of large earthquakes. J. Geophys. Res. 101, 13883-13893 (1996)

30. Gutenberg, B., Richter, C.F.: Frequency of earthquakes in California. Bull. Seismol. Soc. Am. 34, 185-188(1944)

31. Christensen, K., Olami, Z.: Variation of the GutenbergRichter $\mathrm{b}$ values and nontrivial temporal correlations in a spring-block model for earthquakes. J. Geophys. Res. 97, 8729-8735 (1992)

32. Omori, F.: On the aftershocks of earthquakes. J. Coll. Sci. 7, 111-200(1894)

33. Utsu, T., Ogata, Y., Matsuura, R.S.: The centenary of the Omori formula for a decay law of aftershock activity. J. Phys. Earth 43, 1-33 (1995)

34. Reasenberg, P.A., Jones, L.M.: Earthquake hazard after a mainshock in California. Science 243, 1173-1176(1989)

35. Lindman, M., Lund, B., Roberts, R., Jonsdottir, K.: Physics of the Omori law: inferences from interevent time distributions and pore pressure diffusion modelling. Tectonophysics 424, 209-222 (2006) 
36. Chakrabarti, B.K., Stinchcombe, R.B.: Stick-slip statistics for two fractal surfaces: a model for earthquakes. Physica A 270, 27-34 (1999)

37. Yang, D., Yang, P., Zhang, C.: Chaotic characteristic analysis of strong earthquake ground motions. Int. J. Bifurc. Chaos 22, 1250045 (2012)

38. Okubo, P.G., Aki, K.: Fractal geometry in the San Andreas fault system. J. Geophys. Res. 92, 345-355(1987)

39. Sornette, A., Sornette, D.: Self-organized criticality and earthquakes. Europhys. Lett. 9, 197-202 (1989)

40. Shahin, A.M., Ahmed, E., Elgazzar, A.S., Omar, Y.A.: On fractals and fractional calculus motivated by complex systems. arXiv:0901.3618v1 [nlin.AO]

41. Rocco, A., West, B.J.: Fractional calculus and the evolution of fractal phenomena. Physica A 265, 535-546(1999)

42. Samko, S., Kilbas, A., Marichev, O.: Fractional Integrals and Derivatives: Theory and Applications. Gordon and Breach Science, London (1993)

43. Podlubny, I.: Fractional Differential Equations. Academic Press, San Diego (1999)

44. Kilbas, A., Srivastava, H.M., Trujillo, J.: Theory and Applications of Fractional Differential Equations. Elsevier, Amsterdam (2006)

45. Baleanu, D., Diethelm, K., Scalas, E., Trujillo, J.J.: Fractional Calculus: Models and Numerical Methods. Series on Complexity, Nonlinearity and Chaos. World Scientific Publishing, Singapore (2012)

46. Lima, M.F.M., Tenreiro Machado, J.A.: Representation of robotic fractional dynamics in the pseudo phase plane. Acta Mech. Sin. 27, 28-35 (2011)

47. Tenreiro Machado, J.A.: Accessing complexity from genome information. Commun. Nonlinear Sci. Numer. Simul. 17, 2237-2243 (2012)

48. Lima, M.F.M., Tenreiro Machado, J.A., Costa, A.C.: A multidimensional scaling analysis of musical sounds based on pseudo phase plane. Abstr. Appl. Anal. (2012). doi: $10.1155 / 2012 / 436108$
49. Duarte, F.B., Tenreiro Machado, J.A., Duarte, G.M.: Dynamics of the Dow Jones and the Nasdaq stock indexes. Nonlinear Dyn. 61, 691-705 (2010)

50. International Seismological Centre On-line Bulletin, Internatl Seis Cent, Thatcham, United Kingdom (2010). http:// www.isc.ac.uk. Accessed 12 June 2013

51. Young, J.B., Presgrave, B.W., Aichele, H., Wiens, D.A., Flinn, E.A.: The Flinn-Engdahl regionalisation scheme: the 1995 revision. Phys. Earth Planet. Inter. 96, 223-297 (1996)

52. Flinn, E.A., Engdahl, E.R., Hill, A.R.: Seismic and geographical regionalization. Bull. Seismol. Soc. Am. 64, 771993 (1974)

53. Flinn, E.A., Engdahl, E.R.: A proposed basis for geographical and seismic regionalization. Rev. Geophys. 3, 123-149 (1965)

54. http://earthquake.usgs.gov/ Accessed 14 April 2013

55. Tenreiro Machado, J.A., Duarte, G.M., Duarte, F.B.: Identifying economic periods and crisis using the multidimensional scaling. Nonlinear Dyn. 63, 611-622 (2011)

56. Takens, F.: Detecting strange attractors in turbulence. Dyn. Syst. Turbul. 898, 366-381 (1981)

57. International Seismological Centre, On-line Bulletin, http:// www.isc.ac.uk, Internatl. Seis. Cent., Thatcham, United Kingdom, 2011

58. Kostic', S., Franovic', I., Todorovic', K., Vasovic', N.: Friction memory effect in complex dynamics of earthquake model. Nonlinear Dyn. (2013). doi:10.1007/s11071-013-0914-8

59. Ivo Alves, E.: Earthquake forecasting using neural networks: results and future work. Nonlinear Dyn. 44, 341349 (2006)

60. Yas,ar, E.: Variational principles and conservation laws to the Burridge-Knopoff equation. Nonlinear Dyn. 54, 307312 (2008)

61. Gao, J., Hu, J., Tung, W.: Entropy measures for biological signal analyses. Nonlinear Dyn. 68, 431-444 (2012) 